\title{
Medically Important Novel Biomarkers Therapy for Targeting the Cancerous and Tumor Cells in Combating the Infectious Diseases
}

Safdar Iqbal ${ }^{1}$, Abdul Qadeer Saad ${ }^{1}$, Aamal Haleem², Zahida Parveen ${ }^{3}$, Imtiaz Hussain ${ }^{4}$, Muhammad Kashif Qamar ${ }^{5}$, Muhammad Zahid ${ }^{5}$ (

\author{
${ }^{1}$ Department of Zoology, Cholistan University of Veterinary and Animal Sciences, Bahawalpur, Pakistan \\ ${ }^{2}$ Cancer Genetics and Epigenetics Lab, Department of Biosciences, COMSATS University, Islamabad, Pakistan \\ ${ }^{3}$ Department of Biochemistry, University of Agriculture Faisalabad, Pakistan \\ ${ }^{4}$ Department of Zoology, Wildlife and Fisheries, University of Agriculture Faisalabad, Pakistan \\ ${ }^{5}$ Department of Chemistry (Organic Chemistry), Government College University Faisalabad, Pakistan
}

DOI: $10.36348 /$ sjpm.2021.v06i10.013 $\quad$ | Received: 09.09.2021 | Accepted: 18.10.2021 | Published: 27.10 .2021

*Corresponding author: Muhammad Zahid

\section{Abstract}

Cancer biomarkers are the biological molecules produced by the body or tumor in a person with cancer. In order to place the functioning of biomarkers for clinical investigating, they are passed through different clinical trials in order to check their treatment rate as compared to the normal markers. Different genes are involved in causing the breast cancer and early diagnosis through biomarkers provides an effective way to control the mutations caused in cancerous tissues. Genetic biomarkers are those biological molecules that can detect the change in the DNA and RNA structures. HER2 somatic mutations lead to increase in progressions of cancer development non-small cell lung cancers as well as in breast cells. The most important biomarkers are ALF-alpha-fetoprotein is a protein that in humans is encoded by the AFP gene in the liver. Mutational defect in AFP gene leads to severe damage to liver. C-reactive protein (CRP) is the biomarker for inflammation in the body cells. Prostate specific antigen (PSA) is the biomarker used for detection of prostate cancer. Microsatellite instability analysis of a tumor which provides predictive and also prognostic information. Cerebrospinal fluid (CSF) is the important biomarker for the diagnosis of dementia, Alzheimer's disease pathologies.

Keywords: CSF, CRP, AFP, Somatic mutations, cancer development, Cancer biomarkers.

Copyright () 2021 The Author(s): This is an open-access article distributed under the terms of the Creative Commons Attribution 4.0 International License (CC BY-NC 4.0) which permits unrestricted use, distribution, and reproduction in any medium for non-commercial use provided the original author and source are credited.

\section{INTRODUCTION}

Different types of biomarkers have been used for the treatment of various diseases such as cancer, infectious, inflammatory to assist the stage or status of disease $[1,2]$. The advantage of using biomarkers in the fields of medical sciences has become the eminently progressed in the last decades. Cancer biomarkers are the biological molecules produced by the body or tumor in a person with cancer. They usually follow up treatments of clinical diagnosis under medical complications [3, 4]. Cancer biomarkers can be designed in the form of DNA, RNA, protein or metabolomics profiles that binds to the cancerous cells in order to proliferate them in the normal tissues of the body. There are many ways to investigate the designing of the biomarkers used for the treatment of cancer on the basis of DNA sequence, DNA or RNA tests to look for gene fusions or measure RNA or protein levels.
They are tumor targeting once the biological functions of biomarkers compatibly to the particular disease and infectious agent $[5,6]$.

Previously, different drugs and chemical agents have been used to treat the infectious and cancers but they are not are reliable die to high cost and no compatibility to the living system. The most important genetic diseases that causes lethal mutations and difficult to treat. One of such kind of disease id is the gene encoding the enzyme thiopurine methyltransferase in which patients cells fail to metabolize large amounts of the leukemia drug, mercaptopurine that causes severe complications. For such kind of treatment, biomarkers have been designed to clinically point by point treatment of leukemia and other blood borne diseases [7, 8]. 
Safdar Iqbal et al; Saudi J Pathol Microbiol, Oct, 2021; 6(10): 395-400

Table 1: Shows the different types of biomarker and their medical roles

\begin{tabular}{|c|c|c|c|}
\hline $\begin{array}{l}\text { Biomarker/Therapeutic } \\
\text { agent }\end{array}$ & Function & Clinical role & Reference \\
\hline Cancer biomarkers & Clinical diagnosis & $\begin{array}{l}\text { Particular disease and } \\
\text { infectious agent }\end{array}$ & {$[3,4]$} \\
\hline Breast Cancer & HER2 & $\begin{array}{l}\text { Mutations in different tissues } \\
\text { of breast changes }\end{array}$ & $\begin{array}{l}{[3,9,10,} \\
11]\end{array}$ \\
\hline Liver cancer & ALF-alpha-fetoprotein & $\begin{array}{l}\text { Mutational defect in AFP gene } \\
\text { leads to severe damage to liver }\end{array}$ & $\begin{array}{l}{[16,17,} \\
18]\end{array}$ \\
\hline Prostate cancer & Prostate specific antigen & $\begin{array}{l}\text { It appears due to certain } \\
\text { mutational defects in the } \\
\text { prostate gland. }\end{array}$ & {$[17,18]$} \\
\hline Colorectal cancer & KRAS mutations & Abnormal proteins formation & $\begin{array}{l}{[22,23,} \\
24]\end{array}$ \\
\hline Inflammation & C-reactive protein (CRP) & $\begin{array}{l}\text { Biomarker for inflammation in } \\
\text { the body cells }\end{array}$ & {$[27,28]$} \\
\hline Neurological & Cerebrospinal fluid (CSF) & $\begin{array}{l}\text { Important biomarker for the } \\
\text { diagnosis of dementia, } \\
\text { Alzheimer's disease } \\
\text { pathologies. }\end{array}$ & {$[29,30]$} \\
\hline CRISPR/Cas9 system & Genetic defects & Genome editing & {$[8,31,32]$} \\
\hline Gene expression & Expression levels of miRNAs in tumors & Clinical diagnostics & {$[18,19]$} \\
\hline Genetic biomarkers & DNA/RNA & Biological molecules & {$[19,20]$} \\
\hline Immunotherapy & $\begin{array}{l}\text { Best treatments to elucidate the different } \\
\text { responses among different patients }\end{array}$ & Medical treatments & {$[13,14]$} \\
\hline
\end{tabular}

\section{Types of biomarkers and their medical roles}

Breast cancer is one of major cause of deaths among women all around the world due to mutations in different tissues of breast changes to severe form and treatment has become difficult due to risky genes that cause mutated the normal cells. One of such kind of clinical example is the immunohistochemistry for estrogen receptor in breast tumors has been performed for years that become the apparent that many clinical assays do not accurately assess this critical predictive biomarker [3,6]. The other biomarkers are progesterone receptor (PR) and human epidermal growth factor receptor 2 (HER2). In order to place the functioning of biomarkers for clinical investigating, they are passed through different clinical trials in order to check their treatment rate as compared to the normal markers that used for same treatment that have been prohibited due to more side effects as these contained the chemicals agents $[9,10]$.

Different genes are involved that activated as a result of mutations and testing of mutated genes leads effective for controlling the cancers. For example, HER2 gene or for the HER2 protein, samples from the patients required in order to monitor the disease as a result of proteins in the particular gene $[11,12]$. Biomarkers play a critical role in improving the drug development process as well as in the larger biomedical research enterprise. Different genes are involved in causing the breast cancer and early diagnosis through biomarkers provides an effective way to control the mutations caused in cancerous tissues. These biomarkers are frequently associated with a predisposition to cancer and can inform a woman's future risk of developing breast cancer. There is need to design molecular based biomarkers that can target the tumors at molecular level thus helpful for gene expression $[3,6,8]$.

Immunotherapy is one of the best treatments to elucidate the different responses among different patients taken medical treatments. There is difficult to monitor the individual response of each patient. Biomarkers have been used to predict immune response to determine which patients benefit from which type of immunotherapy. Biomarkers are ideal to the immune cells as they can differentiate the cancerous or mutated tissues from normal ones. Biomarkers show responses to abnormal cells once designed for medical therapies. Genetic mutations are a hallmark of malignant tumors and are responsible for the vast majority of cancer's life-threatening characteristics that leads to progression of cancer cells growth at extreme level and life threatening events occurred in most of cases [13-15].

Various biomarkers and their mode of action have been studied in different literature data. The most important biomarkers are ALF-alpha-fetoprotein is a protein that in humans is encoded by the AFP gene. Mutational defect in AFP gene leads to severe damage to liver. It also indicates the working status of liver such as detoxification of chemical toxic compounds from the body. Accumulation of toxic compounds can causes liver toxicity. As a result, liver unable to performs its functions effectively. Hepatocellular carcinoma is the most leading cause of death from cancer rising 
mortality all the word. Biomarkers have been discovered in order to explore the molecular mechanism focused biomarker discovery has also benefited from advances in instrumental design and technology [16$18]$.

\section{Genetic Biomarkers}

Genetic biomarkers are those biological molecules that can detect the change in the DNA and RNA structures. These biomarkers detect abnormalities in the functioning of the substructures of DNA and RNA. DNA-based biomarkers designed to identify genomic mutations, from single DNA building block from base pairs to a large segment of a chromosome that includes multiple genes. RNA-based biomarkers are mostly mRNAs found in tissues and bodily fluids $[1,8]$. Changes in the loop structures of RNA also leads to certain defects and affected the biological process of central dogma. The inhibition of nay enzymes in DNA replication, transcription and translation causes functional abnormal in cells. Discovery of genetic biomarkers helpful to control the genetic diseases arise from mutational defects and other diseases during replicating of the body cells $[19,20]$.

Prostate specific antigen (PSA) is the biomarker used for detection of prostate cancer. PSA is the proteins that are the produced by the normal cells as well as cancerous cells. It appears due to certain mutational defects in the prostate gland. This biomarker may be used not just to diagnose the disease, but to measure its progression and how the treatment is performing [11, 17, 18]. Prostate specific antigen is usually measured by a simple blood test. Since the amount of PSA in the blood is very low, detection of it requires a very sensitive type of technology such as monoclonal antibody technique. There are many factors affecting the blood concentrations of causing the prostate cancer in men such as functioning of prostate gland. More technological advances needed in order to diagnose the PSA proteins at cellular and molecular level with low concentrations of blood samples for testing [21-23].

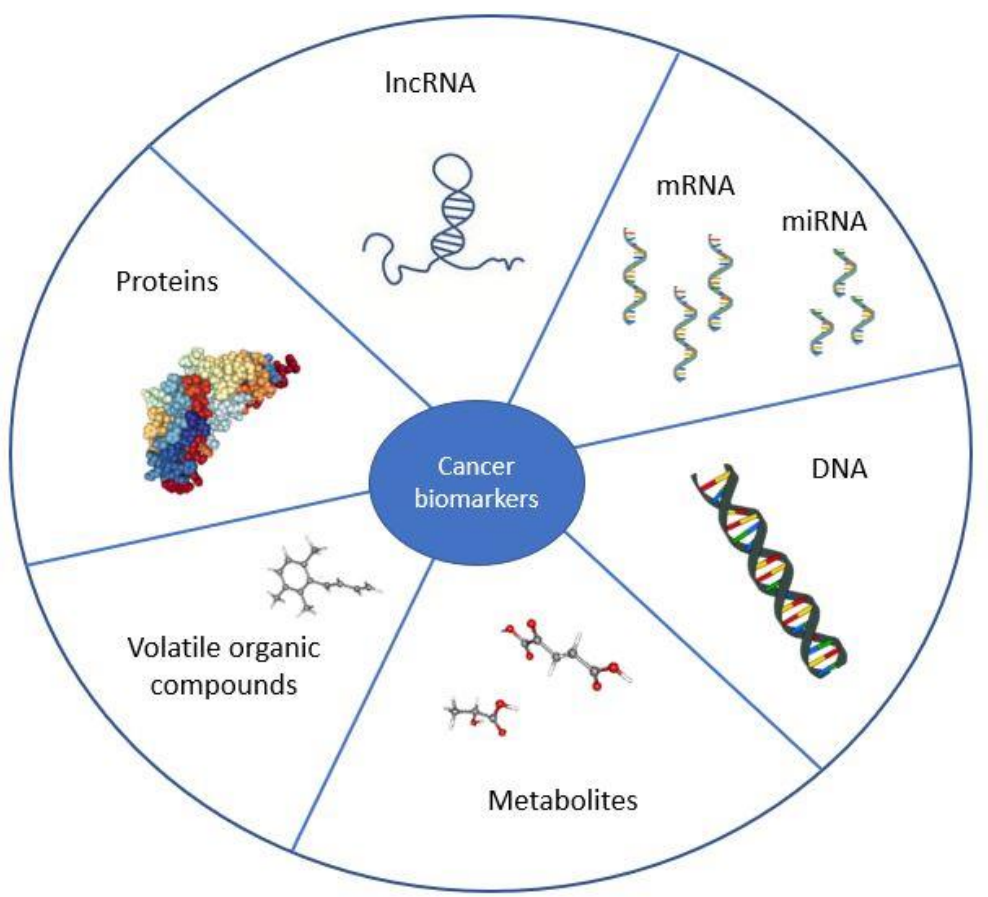

Fig-1: Shows the different types of cancer biomarkers

\section{Other Biomarkers}

Colorectal cancer is one of the major causes of death among human population. The risks of colorectal cancer increasing day by day due to poor diagnosis and malfunction testing of traditional methods. Normally KRAS mutations serve as an information hub for signals which causes colorectal cancer. Microsatellite instability analysis of a tumor which provides predictive and also prognostic information. There are certain gaps in molecular diagnosis of tumors arise during formation of replication and abnormal proteins formation [22-24].
Biomarkers that have been used to control the genetic and infectious diseases, their mode of action is to elucidate the molecular sequences on the cinereous causing agent or abnormal cells arise resulted due to genetic defects. Biomarkers detect the cancerous and other tumor tissues that causes the tumor microenvironment that affects the functionality of normal cells and sometimes unable to grow due to arrest of cell cycle [19-21]. These cellular and molecular events lead to occurring of tumor promoting effects due to the senescence-associated secretory phenotype. Although, biomarkers assist in diagnosis of 
Safdar Iqbal et al; Saudi J Pathol Microbiol, Oct, 2021; 6(10): 395-400

molecular detection of abnormal genes and proteins causing serious defects in normal tissues, more research needed to carry out the target site of occurring of tumor in normal cells. It helps to control the rate of infectious diseases $[25,26]$.

Sometimes, HER2 somatic mutations lead to increase in progressions of cancer development nonsmall cell lung cancers as well as in breast cells. These mutations are involved in activating mutations based on their ability to increase intracellular signaling and ultimately promote the development of tumor growth $[3,8,19]$. Tumor cells can be diagnosed through biopsy and other molecular analysis. It allows the identification of different tissues. Various methods for molecular analysis have been assayed as a procedure called 'tumor cell enrichment. Different analytical methods are employed for extraction of DNA/RNA with tumor cells but their detection sometimes can cause separation of biochemical parameters reigns due to low compatibility. The most important laboratory investigation of the cancerous cells and tissues through histologyagnosticism may be the extreme of a continuous spectrum of action ability, rather than a binary quality [27, 28].

C-reactive protein (CRP) is the biomarker for inflammation in the body cells. It can be performed for liver functioning and other types of severe inflammatory markers such as arthritis. High inflammation showed by C-reactive test as indication of damaging of body cells such as bone, kidneys. A high CRP test result is a sign of acute inflammation. It may be due to serious infection, injury or chronic disease. Sometimes, false positive tests of C-reactive protein (CRP) leads to poor diagnosis that leads to improper medical treatment. Therefore, other molecular markers needed to discovered for accessing the abnormalities such as inflammations. Cerebrospinal fluid (CSF) is the important biomarker for the diagnosis of dementia, Alzheimer's disease pathologies. Detection of the cerebrospinal fluid helpful to control the neurodegenerative disorders [29, 30].

\section{Role of CRISPR/Cas9 and other systems}

The CRISPR/Cas9 system is one of the most important revolutionary advancements in the fields of molecular biology and genetics that depends upon the ribonucleotide complex formation instead of DNA recognition. It depends upon on the genome editing of the specific fragment or defective part of the nuclear region [8]. It can be passed through series of events show that mutational defects and other genetic abnormalities can be easily manipulated through CRISPR/Cas9 system. Genome engineering technology has provided researchers with an invaluable tool to accelerate the generation of mouse models for biomedical. Many of the infectious diseases caused by bacteria, viruses and other pathogens have been controlled through CRISPR technology with particular focused on genomic sequences to be editing in order to replace the defective of nonfictional part of the nuclear regions. In this way, it can be used to detect and destroy DNA from similar bacteriophages during subsequent infection $[31,32]$.

There are different methods for checking the expression levels of miRNAs in tumors. By measuring the concentrations of miRNA, it becomes easy to treat the genetic defects. This property of the cancer cell is being utilized in cancer diagnosis for early and accurate cancer diagnosis. P53 gene mutation is prevalent in several cancers. Different drugs have been used to control the mutations in P53 gene in order to arrest the cell cycle $[18,19]$. One of such kind of drug that has been effectively used is the gendicine induces the expression of p53 restores its activity and destroys the tumor cells. Although, advancements in genetic basis of cancers have been made to target the genetic mutations, but through genetic engineering and combinations of other biomedical sciences helpful as a bridge for targeting tumors and cancerous cells proliferation. In this way, Alzheimer and neurodegenerative disorders can be treated in better ways [33-35].

\section{CONCLUSION}

The expression of the different genes can be taken into consideration through revolutionary advancements in macular biology. Biomarkers can be also associated with some degree of risk. Molecular alterations that have been used for the cancer diagnosis are occurring at the DNA level include gene replication, rearrangements or translocations, point mutations, deletions or insertions. Genomic editing through CRISPR technology as the most powerful system to treat the number of diseases.

\section{REFERENCES}

1. Langan, R. C., Mullinax, J. E., Raiji, M. T., Upham, T., Summers, T., Stojadinovic, A., \& Avital, I. (2013). Colorectal cancer biomarkers and the potential role of cancer stem cells. Journal of Cancer, 4(3), 241.

2. Van Tilborg, A. A., Bangma, C. H., \& Zwarthoff, E. C. (2009). Bladder cancer biomarkers and their role in surveillance and screening. International Journal of Urology, 16(1), 23-30.

3. Brinkman, B. M. (2004). Splice variants as cancer biomarkers. Clinical biochemistry, 37(7), 584-594.

4. Chervona, Y., \& Costa, M. (2012). Histone modifications and cancer: biomarkers of prognosis?.American journal of cancer research, 2(5), 589.

5. Thind, A., \& Wilson, C. (2016). Exosomal miRNAs as cancer biomarkers and therapeutic targets. Journal of extracellular vesicles, 5(1), 31292. 
Safdar Iqbal et al; Saudi J Pathol Microbiol, Oct, 2021; 6(10): 395-400

6. Simpson, R. W., Berman, M. A., Foulis, P. R. Divaris, D. X., Birdsong, G. G., Mirza, J., ... \& Fitzgibbons, P. L. (2015). Cancer biomarkers: the role of structured data reporting. Archives of Pathology and Laboratory Medicine, 139(5), 587593.

7. Arnaiz, E., Sole, C., Manterola, L., Iparraguirre, L., Otaegui, D., \& Lawrie, C. H. (2019, October). CircRNAs and cancer: biomarkers and master regulators. In Seminars in cancer biology (Vol. 58, pp. 90-99). Academic Press.

8. Ansari, D., Aronsson, L., Sasor, A., Welinder, C., Rezeli, M., Marko-Varga, G., \& Andersson, R. (2014). The role of quantitative mass spectrometry in the discovery of pancreatic cancer biomarkers for translational science. Journal of translational medicine, 12(1), 1-15.

9. Hasan, S., Jacob, R., Manne, U., \& Paluri, R. (2019). Advances in pancreatic cancer biomarkers. Oncology reviews, 13(1).

10. Brambilla, C., Fievet, F., Jeanmart, M., de Fraipont, F., Lantuejoul, S., Frappat, V., ... \& Moro-Sibilot, D. (2003). Early detection of lung cancer: role of biomarkers. European Respiratory Journal, 21(39 suppl), 36s-44s.

11. Pellino, G., Gallo, G., Pallante, P., Capasso, R., De Stefano, A., Maretto, I., ... \& Selvaggi, F. (2018). Noninvasive biomarkers of colorectal cancer: role in diagnosis and personalised treatment perspectives. Gastroenterology research and practice, 2018.

12. Marrugo-Ramírez, J., Mir, M., \& Samitier, J. (2018). Blood-based cancer biomarkers in liquid biopsy: a promising non-invasive alternative to tissue biopsy. International journal of molecular sciences, 19(10), 2877.

13. Picchio, M., Mapelli, P., Panebianco, V., Castellucci, P., Incerti, E., Briganti, A., ... \& Fanti, S. (2015). Imaging biomarkers in prostate cancer: role of PET/CT and MRI. European journal of nuclear medicine and molecular imaging, 42(4), 644-655.

14. Jin, H., Wu, Y., \& Tan, X. (2017). The role of pancreatic cancer-derived exosomes in cancer progress and their potential application as biomarkers. Clinical and Translational Oncology, 19(8), 921-930.

15. Cardinale, D., Salvatici, M., \& Sandri, M. T. (2011). Role of biomarkers in cardioncology. Clinical Chemistry and Laboratory Medicine (CCLM), 49(12), 1937-1948.

16. Guinde, Julien, Diane Frankel, Sophie Perrin, Valérie Delecourt, Nicolas Lévy, Fabrice Barlesi, Philippe Astoul, Patrice Roll, and Elise Kaspi. "Lamins in lung cancer: Biomarkers and key factors for disease progression through miR-9 regulation?." Cells 7, no. 7 (2018): 78.
17. Fu, S. W., Chen, L., \& Man, Y. G. (2011). miRNA biomarkers in breast cancer detection and management. Journal of Cancer, 2, 116.

18. Tan, L. L., \& Lyon, A. R. (2018). Role of biomarkers in prediction of cardiotoxicity during cancer treatment. Current treatment options in cardiovascular medicine, 20(7), 1-14.

19. da Silva Oliveira, K. C., Araújo, T. M. T., Albuquerque, C. I., Barata, G. A., Gigek, C. O., Leal, M. F., ... \& Calcagno, D. Q. (2016). Role of miRNAs and their potential to be useful as diagnostic and prognostic biomarkers in gastric cancer. World journal of gastroenterology, 22(35), 7951.

20. Duffy, M. J. (2015). Personalized treatment for patients with colorectal cancer: role of biomarkers. Biomarkers in medicine, 9(4), 337347.

21. Lee, J. D., Cai, Q., Shu, X. O., \& Nechuta, S. J. (2017). The role of biomarkers of oxidative stress in breast cancer risk and prognosis: a systematic review of the epidemiologic literature. Journal of Women's Health, 26(5), 467-482.

22. Srivastava, A., \& Creek, D. J. (2019). Discovery and validation of clinical biomarkers of cancer: a review combining metabolomics and proteomics. Proteomics, 19(10), 1700448.

23. Fu, L. L., Li, C. J., Xu, Y., Li, L. Y., Zhou, X., Li, D. D., ... \& Zheng, L. W. (2017). Role of lncRNAs as novel biomarkers and therapeutic targets in ovarian cancer. Critical Reviews ${ }^{\mathrm{TM}}$ in Eukaryotic Gene Expression, 27(2).

24. Aslam, M. I., Taylor, K., Pringle, J. H., \& Jameson, J. S. (2009). MicroRNAs are novel biomarkers of colorectal cancer. Journal of British Surgery, 96(7), 702-710.

25. Zarogoulidis, P., Tsakiridis, K., Karapantzou, C., Lampaki, S., Kioumis, I., Pitsiou, G., ... \& Zarogoulidis, K. (2015). Use of proteins as biomarkers and their role in carcinogenesis. Journal of Cancer, 6(1), 9.

26. Bădilă, E. L. I. S. A. B. E. T. A., Japie, C., \& Bartoş, D. A. N. I. E. L. A. (2014). Cancer biomarkers in clinical practice. Rom J Intern Med, 52(4), 223-232.

27. Santosh, A. B. R., Jones, T., \& Harvey, J. (2016). A review on oral cancer biomarkers: Understanding the past and learning from the present. Journal of cancer research and therapeutics, 12(2), 486.

28. Shau, H., Chandler, G. S., Whitelegge, J. P., Gornbein, J. A., Faull, K. F., \& Chang, H. R. (2003). Proteomic profiling of cancer biomarkers. Briefings in Functional Genomics, 2(2), 147-158.

29. Qu, H., Xu, W., Huang, Y., \& Yang, S. (2011). Circulating miRNAs: promising biomarkers of human cancer. Asian Pac J Cancer Prev, 12(5), 1117-1125. 
Safdar Iqbal et al; Saudi J Pathol Microbiol, Oct, 2021; 6(10): 395-400

30. Ye, F., Zhao, Y., El-Sayed, R., Muhammed, M., \& Hassan, M. (2018). Advances in nanotechnology for cancer biomarkers. Nano Today, 18, 103-123.

31. Bag, A., Jyala, N. S., \& Bag, N. (2015). Cytochrome P450 1A1 genetic polymorphisms as cancer biomarkers. Indian journal of cancer, 52(4), 479.

32. Pudil, R., Mueller, C., Čelutkienè, J., Henriksen, P. A., Lenihan, D., Dent, S., ... \& Lyon, A. R. (2020). Role of serum biomarkers in cancer patients receiving cardiotoxic cancer therapies: a position statement from the Cardio-Oncology Study Group of the Heart Failure Association and the CardioOncology Council of the European Society of
Cardiology. European journal of heart failure, 22(11), 1966-1983.

33. Kashyap, M. K., Marimuthu, A., Kishore, C. J. H., Peri, S., Keerthikumar, S., Prasad, T. S. K., ... \& Pandey, A. (2009). Genomewide mRNA profiling of esophageal squamous cell carcinoma for identification of cancer biomarkers. Cancer biology \& therapy, 8(1), 36-46.

34. Bhatt, A. N., Mathur, R., Farooque, A., Verma, A., \& Dwarakanath, B. S. (2010). Cancer biomarkerscurrent perspectives. Indian J Med Res, 132(2), 129-149.

35. Villalobos, P., \& Wistuba, I. I. (2017). Lung cancer biomarkers. Hematology/Oncology Clinics, 31(1), 13-29. 